\title{
A Simulation Study on the Effect of Cavity Shapes on the Penetration of Linear Shaped Charges with Curved Liners
}

\author{
S. K. Dong, ${ }^{a}$ A. Y. Cui, ${ }^{a}$ H. K. Wei, ${ }^{a}$ X. D. Huang, ${ }^{b}$ Q. Liu, ${ }^{b}$ and G. X. Pei ${ }^{b}$ \\ ${ }^{a}$ Naval Aeronautical University Qingdao Campus, Qingdao, China \\ b North Special Energy Group Xi'an Qinghua Company, Xi'an, China
}

The finite element models of curved-liner shaped charges penetrating aluminum-alloy thin plates were constructed with the ANSYS/LS-DYNA software to analyze the effect of cavity shapes on the charge penetration with curved liners. The cavity height, radius, and spatial dimensions were numerically simulated. Simulation results indicate that these properties are important factors in determining the penetration of curved-liner charges. The penetration depth exhibits an initial increase followed by a decrease with the fixed cavity radius. When the cavity height is fixed, the penetration depth decreases with the cavity radius. When the cavity is semicircular, the penetration depth first increases and then decreases with the cavity radius and height.

Keywords: linear shaped charge, cavity shape, curved liner, penetration.

Introduction. Linear shaped charges that have already been widely applied in military and civilian areas have outstanding features like convenience, effectiveness and flexibility because of the advantage of the Munroe effect. The work reliability is highly required since the ignition environment of these charges is quite specific. Hence, their charge amounts are usually redundant with relatively high energy margin. In recent years, the application fields of linear shaped charges keep expanding with the continuous perfection of explosive cutting technology, and charge detonation near people is required in more and more conditions [1]. Some of the examples are the precise blasting system of airliner cabin door [2], the flexible cutter of bus windshields [3], the canopy-ejection system of a fighter [4], the explosive cutting device of emergency escape route [5], etc. These linear shaped charges have small dimensions, low charge amounts, and limited energy levels, so their accuracy and fineness are more emphasized during the design and utilization of such LSCs. Thus, the power of such LSCs should be carefully designed under the premise of finishing the cutting task in order to reduce the damages to surrounding people, equipment, instrument, and structure.

The available studies were mainly focused on wedge-shaped liners as for the liner shape of LSCs. Besides the wedged liner, another typical liner of LSCs is the curved liner. Theoretical analyses [6] and experimental studies [7] have demonstrated that the advantage of the curved liner is its larger jet mass. It has been found that the cutting depth of LSC with a curved liner exceeds that of the same charge amount with a wedged liner. However, there are very few studies on linear shaped charges with curved liners, while the effect of cavity shapes on the penetration of LSCs with curved liners is not reported yet. Therefore, curved-liner LSCs of various cavity shapes are analyzed based on the numerical simulation in this study, which is aimed at the refinement of the linear shaped charge theory and substantiation of the design of curved-liner LSCs.

1. Shape Parameters of Cavity. Figure 1 is the cross section of a curved-liner LSC. The shaded part in the figure is the metal shell. High-energy explosives are filled inside the shell. Metals in one side of the cavity play the role of the liner. After the charge is detonated, a detonation wave will propagate through the explosive core. When the detonation wave reaches the liner, the blasting products will act on the liner with extremely high pressure. Then, the liner start to collapse along the axis of symmetry and finally forms 
a high-speed metal jet [8]. The existence of cavity results in the high energy density and strong directivity of the jet. Thus, the penetration performance is significantly improved, as compared to solid charges with no cavity.

The structural parameters of the cross section of curved-liner LSCs are: external radius of metal shell $R$, thickness of metal shell $D_{1}$, thickness of liner $D_{2}$, radius of cavity $r$, and height of cavity $h$. The values of $R, D_{1}$, and $D_{2}$ are firstly determined, according to the mechanical properties of desired cut targets for designing LSCs with curved liners. Further, the design of cavity shape determines whether the energy in the linear shaped charge can be fully utilized or not via the Munroe effect.

To investigate the influence of cavity shape parameters on the jet penetration performance, the finite element models of LSC penetrating thin metal plates are developed by using ANSYS/LS-DYNA and LS-PREPOST. For the convenience of calculation, it is assumed in this paper that the metal shell external radius $R=2 \mathrm{~mm}$, metal shell thickness $D_{1}=0.2 \mathrm{~mm}$, and liner thickness $D_{2}=0.1 \mathrm{~mm}$.

2. Elaboration of Finite Element Models. The process of linear shaped charge penetrating a target plate is a spatial mechanics issue. However, if the LSC is considered to detonate with a linear initiation pattern, the complicated $3 \mathrm{D}$ issue can be reduced to the $2 \mathrm{D}$ planar one. As the LSC cross section is an axial-symmetric structure, and the charge dimension is far smaller than the target plate, both the target plate and LSC can be considered as axisymmetric domains and only half of the model is needed.

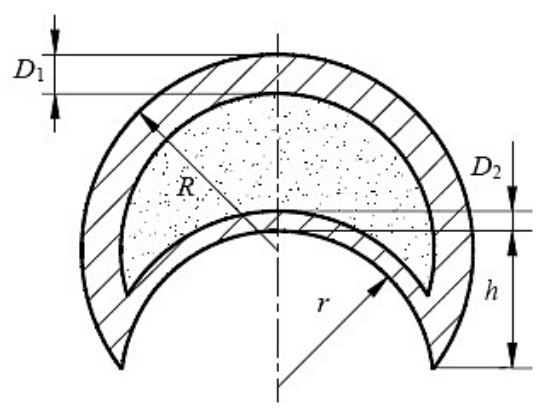

Fig. 1. Cross section of curved-liner LSC.

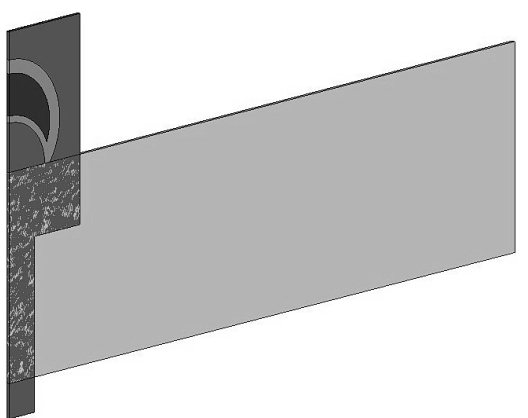

Fig. 2. Schematics of the finite element model.

The process of lead-sheathed LSCs filled with RDX penetrating $3 \mathrm{~mm}$ aluminum alloy plates is taken as the engineering background of this study. The model consists of RDX, lead shell, air, and aluminum alloy plate. To simplify the calculation procedure, a single layer solid mesh is used to create four PARTs, including explosive charge, shell, space medium, and target plate, as shown in Fig. 2. The target plate is defined as PART 1, while the explosive charge, shell and spatial medium are defined as PART 2. The PART 1 is under the explosive effect of PART 2. The $\mathrm{cm}-\mathrm{g}-\mu$ s unit system is used within the model.

The material models and state equations of each part are listed in Table 1. Material parameters used in the simulation are: for RDX, density, $1.45 \mathrm{~g} / \mathrm{cm}^{3}$, blasting speed, 7600 $\mathrm{m} / \mathrm{s}$, in its state equation $A=611.3 \mathrm{GPa}, B=10.65 \mathrm{GPa}, R_{1}=4.4, R_{2}=1.2$, and $\omega=0.32$; for the lead shell, density, $11.3 \mathrm{~g} / \mathrm{cm}^{3}$, shear modulus, $7 \mathrm{GPa}$, in its state equation $S_{1}=1.429, R_{1}=4.4, S_{2}=S_{3}=0, \gamma_{0}=2.77, a=0$; for the air, density, $1.25 \mathrm{~kg} / \mathrm{m}^{3}$, in its state equation $S_{1}=S_{2}=S_{3}=0, \gamma_{0}=1.4, a=0$; for the aluminum alloy plate, density, $2.78 \mathrm{~g} / \mathrm{cm}^{3}$, elastic modulus, $71 \mathrm{GPa}$, Poisson's ratio, 0.32 , and yield stress, $380 \mathrm{MPa}$.

\section{Results and Discussion.}

3.1. Cavity Height Effect on the Penetration. Firstly, the penetration differences are compared when cavity height $h$ varies. Based on the control variable method, various parameters are assumed as target plate thickness, $3 \mathrm{~mm}$, and the cavity radius $r=0.070 \mathrm{~mm}$. 
$\mathrm{T}$ a b 1 e 1

Material Models and State Equations of Each Part

\begin{tabular}{||c|c|c|c||}
\hline \hline Component & Material & Material model & State equation \\
\hline Explosive & RDX & HIGH_EXPLOSIVE_BURN & J-W-L \\
\hline Shell & Lead & JOHNSON_COOK & GRUNEISEN \\
\hline Medium & Air & Null & CRUNEISEN \\
\hline Target plate & Aluminum alloy & PLASTIC_KINEMATIC & - \\
\hline
\end{tabular}

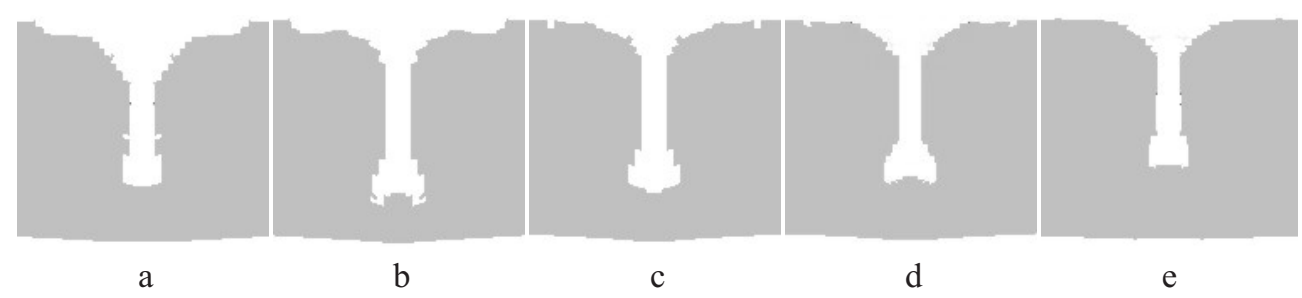

Fig. 3. Comparison of penetration performances under different cavity heights: (a) $h=0.060 \mathrm{~mm}$; (b) $h=0.065 \mathrm{~mm}$; (c) $h=0.070 \mathrm{~mm}$; (d) $h=0.075 \mathrm{~mm}$; (e) $h=0.080 \mathrm{~mm}$.

When a new type of LSC is designed, the cavity height is approximately equal to the cavity radius. The approximate equality can guarantee the full utilization of the Munroe effect of shaped charge. In this subsection, cavity height values of $0.060,0.065,0.070,0.075$, and $0.080 \mathrm{~mm}$ were used. The kerf area is selected as the main observation zone. The results of five kinds of LSCs penetrating target plates are shown in Fig. 3.

It is implied that the power of all five types of LSCs is not strong enough to cut off aluminum alloys of $3 \mathrm{~mm}$ thickness. It can be observed from the fracture morphologies of aluminum plates that the kerf has a trumpet shape, and its width is sharply reduced within 1 $\mathrm{mm}$ along the plate thickness direction. The kerf bottom width increases slightly, especially for LSCs of $h, 0.065,0.070$, and $0.075 \mathrm{~mm}$. The kerf bottom shows an obvious bursiform, which indicates that the successive jet can no longer burst through residues in front of it in the terminal stage of penetration. Thus, the bulk deposition of the metal jet in the kerf terminal takes place, and the kerf width expands.

As shown in Fig. 4, the penetration depth $d$ is defined as the distance between the upper surface of plate and the bottommost of the kerf center line. Figure 5 is obtained by plotting the cavity height $h$, and the corresponding penetration depth, $d$, in the coordinate system.

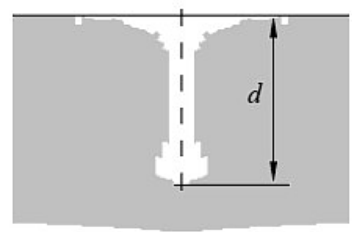

Fig. 4. Definition of penetration depth.

As shown in Fig. 5, when the cavity radius $r$ equals to $0.070 \mathrm{~mm}$, the maximum penetration depth of the five LSCs can reach $2.325 \mathrm{~mm}$, which corresponds to a cavity height of $0.070 \mathrm{~mm}$; the minimum penetration depth is $1.975 \mathrm{~mm}$ under a cavity height of $0.080 \mathrm{~mm}$. Therefore, the penetration depth, $d$, presents a variation tendency of growth followed by drop with an increase in $h$. When $h$ is less than $0.070 \mathrm{~mm}$, with the increase 


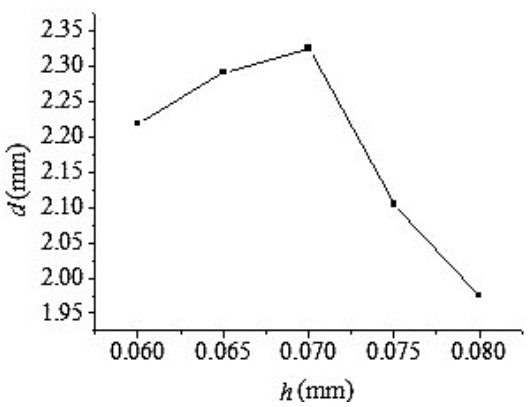

Fig. 5. Relationship between cavity height and penetration depth.

of $h, d$ rises and reaches its peak value at $h=0.070 \mathrm{~mm}$. If $h$ continues to grow, the penetration depth $d$ sharply drops, and the cutting performance of LSCs is sharply reduced. This can be attributed to the fact that an increase in $h$ results in the sufficient tension space for the formation of jet when $h$ is less than $0.070 \mathrm{~mm}$. Thus, more liner microunits can take part in the jet formation. However, when $h$ exceeds $0.070 \mathrm{~mm}$, the increase in $h$ fails to compensate the reduction of jet kinetic energy caused by the charge amount reduction, though it can make the jet expand more sufficiently. Hence, the power of LSCs is obviously reduced.

3.2. Cavity Radius Effect on the Penetration. The cavity radius effect on the cutting performance of LSCs is discussed for the constant cavity height $h$. It is assumed that the target plate thickness is $3 \mathrm{~mm}$, while $h$ is equal to $0.070 \mathrm{~mm}$. The cavity radii for five LSCs are determined as $0.060,0.065,0.070,0.075$, and $0.080 \mathrm{~mm}$, according to the approximate-equality principle of cavity height and radius. The results of five LSCs penetrating target plates are shown in Fig. 6.

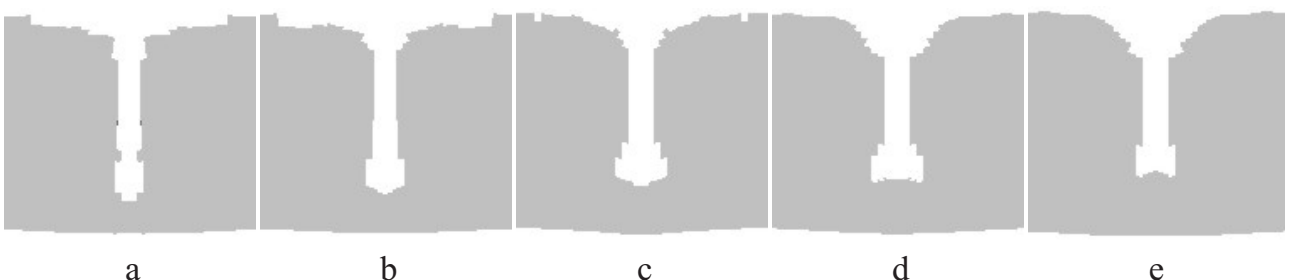

Fig. 6. Comparison of penetration performances under different cavity radii: (a) $r=0.060 \mathrm{~mm}$; (b) $r=0.065 \mathrm{~mm}$; (c) $r=0.070 \mathrm{~mm}$; (d) $r=0.075 \mathrm{~mm}$; (e) $r=0.080 \mathrm{~mm}$.

According to the simulation results, the LSC with a cavity radius of $0.060 \mathrm{~mm}$ has the highest penetration depth among the five shaped charges. Its kerf width is uniform, which indicates that the convergence effect of metal jet is favorable under this parameter condition and the energy density is high. Besides, the penetration depth $d$ shows a decreasing tendency with the increase of $r$. To further analyze the relationship between $d$ and $r$, the penetration depth values of five kinds of LSCs are evaluated by using LS-PREPOST software. Figure 7 is obtained by plotting the cavity radius $r$ and the corresponding penetration depth $d$ in the coordinate system.

It is clear that the penetration performance of LSCs decreases with the increase of cavity radius for $0.070 \mathrm{~mm}$ of $h$. The penetration depth is $2.550 \mathrm{~mm}$ when $r$ is equal to $0.060 \mathrm{~mm}$, while the penetration depth drops to $2.107 \mathrm{~mm}$ when $r$ rises to $0.080 \mathrm{~mm}$. The results can be explained as follows. Although the increase of $r$ can enlarge the cavity under a constant $h$, it cannot provide enough space for the longitudinal growth of jet. Thus, the jet formation process is restricted. At the same time, the charge amount is slightly decreased 


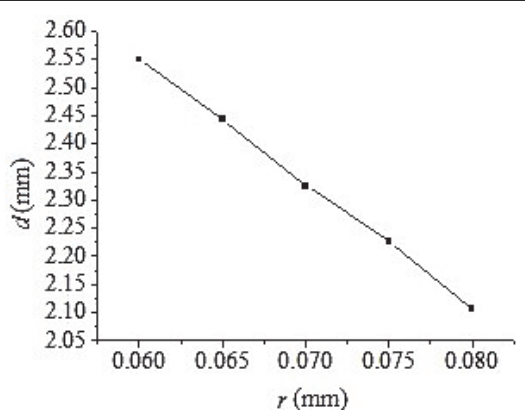

Fig. 7. Relationship between cavity radius and penetration depth.

and the energy transferred to the liner body decreases accordingly. Besides, as shown in Fig. 7, an obvious linear relationship can be found between the penetration depth $\mathrm{d}$ and cavity radius $r$ in the LSCs with a cavity height of $0.070 \mathrm{~mm}$. In details, when the cavity height increases by $0.01 \mathrm{~mm}$, the penetration depth decreases by $0.11 \mathrm{~mm}$.

3.3. Cavity Spatial Dimension Effect on the Penetration. Finally, the effect of cavity spatial dimension on the penetration is discussed. For the convenience of study, height $h$, is taken equal to radius $r$, and circle-center of cavity is located at the intersection point of the two-end connecting line and symmetry axis. Under such circumstances, the cavity is just a semicircle. The results of five LSCs (with both the cavity radius $r$ and cavity height $h$ of $0.040,0.050,0.060,0.070$, and $0.080 \mathrm{~mm}$ ) penetrating aluminum alloy plates of $3 \mathrm{~mm}$ thickness are shown in Fig. 8. It is obvious that these five values above are not enough to cover all the conditions, but they are still significant and meaningful in exploring the influential rules of cavity spatial dimension on the penetration performance.

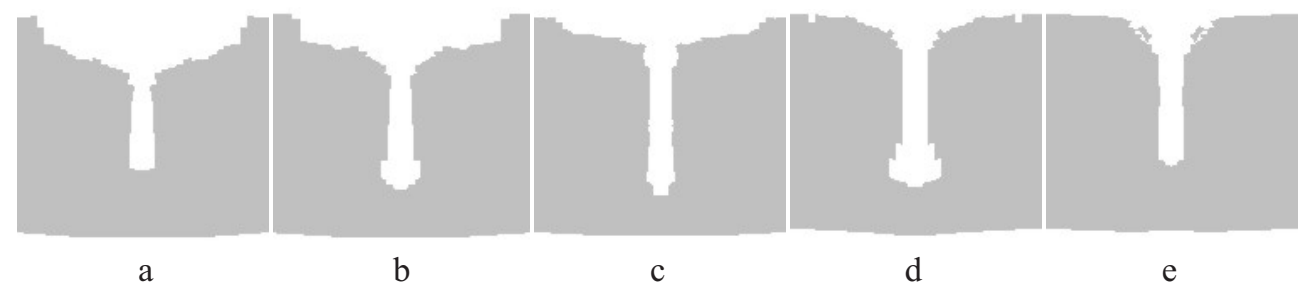

Fig. 8. Comparison of penetration performances under different cavity spatial dimensions: (a) $h=$ $=r=0.040 \mathrm{~mm}$; (b) $h=r=0.050 \mathrm{~mm}$; (c) $h=r=0.060 \mathrm{~mm}$; (d) $h=r=0.070 \mathrm{~mm}$; (e) $h=r=$ $=0.080 \mathrm{~mm}$.

By comparing the penetration results of above LSCs, it can be found that when $h=r=0.040,0.050$, and $0.060 \mathrm{~mm}$, the area eroded by the jet is relatively larger than the other two LSCs. Hence, the metals on both sides of the cavity bottom do not involve in the formation of jet, but they can still bring damage to the target plate. When $h=r=0.050$ and $0.070 \mathrm{~mm}$, the kerf bottom shows an obvious pocket-shape morphology. This is attributed to the bulk deposition of metal residues around the impact point between jet and target. In details, though successive jet still has a speed higher than the critical penetration speed, it can no longer burst through these thick and heavy residues and have to flow to two sides of the kerf. Figure 9 can be obtained by plotting the penetration depth $d$ under different parameters.

Similar to Fig. 5, the penetration depth $d$ initially increases and then decreases with increasing cavity height $h$ and cavity radius $r$. When $h=r=0.040 \mathrm{~mm}$, the liner micro-units involving the formation of the jet are too less, and the Munroe effect cannot be fully reflected. As a consequence, the energy of LSCs cannot be fully used and the penetration effect is unfavourable. Afterwards, the Munroe effect becomes more obvious 


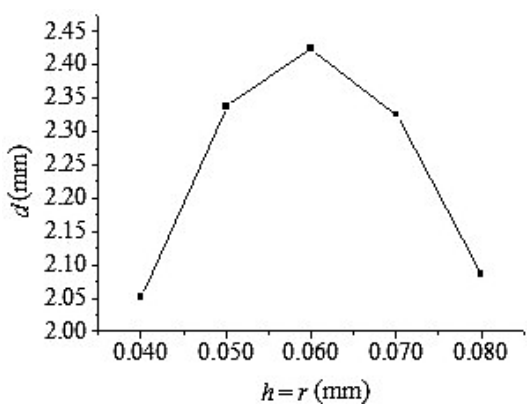

Fig. 9. Relationship between cavity spatial dimension and penetration depth.

and the penetration performance of LSCs continuously increases if the cavity space is suitably expanded. After the maximum penetration value reaches $2.424 \mathrm{~mm}$ at $h=r=$ $=0.060 \mathrm{~mm}$, the energy carried by the LSC is reduced and its power is weakened if the spatial dimension of cavity continues to grow. The reason is that the reduction of charge amount cannot be avoided although the mass of metal jet penetrating the target plate increases to some extent.

Conclusions. It is demonstrated that the height, radius, and spatial dimension of cavity are important parameters determining the penetration performance including penetration depth and kerf morphology of linear shaped charges with curved liners. During the design of LSCs, the three parameters should be adjusted according to requirements of application so as to optimize the shape parameters of LSCs.

When the radius of cavity is a fixed value, the penetration depth presents a first-increase-then-decrease tendency with the increase of cavity height. The penetration depth decreases with the increase of cavity radius when the cavity height is kept constant. Besides, when the cavity shape is semicircular, the penetration depth firstly increases and then drops with the cavity spatial dimension.

1. H. Y. Ma, Y. Long, H. Y. Lu, et al., "Damage patterns of aluminum alloy under the shock of linear shaped charge cutter's explosion," Init. Pyrotech., 32, No. 3, 20-24 (2010).

2. Y. H. Wang, W. Xiong, Z. F. Chen, et al., "Research on the precise measurement of linear charge density of linear shaped charge," Init. Pyrotech., 34, No. 5, 4-8 (2012).

3. S. H. Zhang and R. J. Gou, "Study on destruction of laminated glass under the impact of shaped charge," Init. Pyrotech., 38, No. 3, 17-21 (2016).

4. B. H. Yuan, K. Hu, C. Xiao, et al., "Explosive cutting of PMMA," Chinese J. Expl. Propell., 26, No. 3, 70-72 (2003).

5. W. C. Feng, Aviation Emergency Escape System [in Chinese], National Defense Industry Press, Beijing (2014).

6. S. L. Yan and Y. J. Wang, "Theoretical study on circular cover linear charge," Eng. Blast., 8, No. 2, 6-9 (2002).

7. Y. J. Wang, S. L. Yan, and K. L. Hu, "Comparison of curved-liner and wedged-liner of linear shaped charge," Coal Mine Blast., 18, No. 4, 3-6 (2001).

8. D. Novotney and M. Mallery, "Historical development of linear shaped charge," in: 43rd AIAA/ASME/SAE/ASEE Joint Propulsion Conference \& Exhibit (July 8-11, 2007, Cincinnati, OH), AIAA 2007-5141 (2007), DOI: 10.2514/6.2007-5141. 